\title{
Source size measurement options for low-emittance light sources
}

\author{
N. Samadiø, ${ }^{1, *}$ X. Shi,$^{2, \dagger}$ L. Dallin, ${ }^{3}$ and D. Chapman $\odot^{4}$ \\ ${ }^{1}$ Physics and Engineering Physics, University of Saskatchewan, 116 Science Place, \\ Saskatoon, Saskatchewan, S7N5E2, Canada \\ ${ }^{2}$ Advanced Photon Source, Argonne National Laboratory, \\ 9700 South Cass Avenue, Lemont, Illinois 60439, USA \\ ${ }^{3}$ Canadian Light Source, 44 Innovation Boulevard, Saskatoon, Saskatchewan, S7N2V3, Canada \\ ${ }^{4}$ Anatomy and Cell Biology, University of Saskatchewan, 107 Wiggins Road, \\ Saskatoon, Saskatchewan, S7N5E5, Canada
}

(Received 11 October 2019; accepted 27 January 2020; published 21 February 2020)

\begin{abstract}
Radiation-based techniques for measuring electron source sizes are widely used as emittance diagnostics at existing synchrotron sources. Three of these techniques, namely, pinhole imaging, double-slit interferometry, and a K-edge filter-based beam position and size monitor system (ps-BPM), are evaluated for measuring source sizes at low-emittance storage rings. Each technique is reviewed with a detailed system description, design optimization, and practical considerations targeted for small source sizes. Pinhole imaging has the simplest setup and gives the beam profile in both transverse dimensions but with limited resolution. Double-slit interferometry has the highest resolution but with a limited detectable size range. The ps-BPM system shows reasonable resolution for monitoring small source sizes and divergence and can give real-time information of the source position and angle. New facilities may consider an integrated system that combines some or all of these techniques.
\end{abstract}

DOI: 10.1103/PhysRevAccelBeams.23.024801

\section{INTRODUCTION}

Accurate measurements of electron source emittance [1] are increasingly gaining attention as new-generation synchrotron sources are being designed and built. These new machines are mostly based on multibend achromat (MBA) lattices [2] to achieve ultrasmall emittance in the $\mathrm{pm} \cdot \mathrm{rad}$ level $[3,4]$. As a result, improved or new diagnostic tools are desired to be able to measure and monitor such small emittances.

Electron source emittance is usually obtained indirectly from the source size and/or divergence measurements combined with the knowledge of machine parameters [5]. The most commonly used methods to measure and monitor the electron source size are pinhole imaging [6-8] and double-slit interferometry [9-11]. The pinhole imaging technique is widely used in the x-ray regime at many synchrotron facilities because of its focus-free feature. The

\footnotetext{
*orresponding author. nazanin.samadi@usask.ca

Corresponding author. xshi@anl.gov

Published by the American Physical Society under the terms of the Creative Commons Attribution 4.0 International license. Further distribution of this work must maintain attribution to the author(s) and the published article's title, journal citation, and DOI.
}

double-slit interferometry method is based on the measurement of the transverse coherence of the photon beam and has a high resolution (ability to measure small source sizes). The double-slit interferometry can be used in both $\mathrm{x}$-ray and visible light regimes, but most of the existing systems are using visible light. Recently, a phase-space beam position and size monitor (ps-BPM) system was developed for the full characterization of the electron source position, angle, size, and divergence $[12,13]$. It was shown to have potential in measuring small source sizes as well [14]. In this paper, we will focus on the review of these three methods and discuss the general principles, insights on the optimization of each system, their advantages and limitations, and considerations for building practical devices.

\section{PINHOLE IMAGING}

\section{A. System description}

Pinhole imaging is the most common way of measuring the transverse profile of the electron source at thirdgeneration light sources [6-8]. A schematic of an x-ray pinhole imaging system is shown in Fig. 1. A typical system consists of a pinhole located at a distance $p$ downstream of the source and a detector system at a distance $q$ downstream of the pinhole.

The image profile recorded on the detector contains contributions from the magnified source image, the point spread function (PSF) of the pinhole, and the PSF of the 


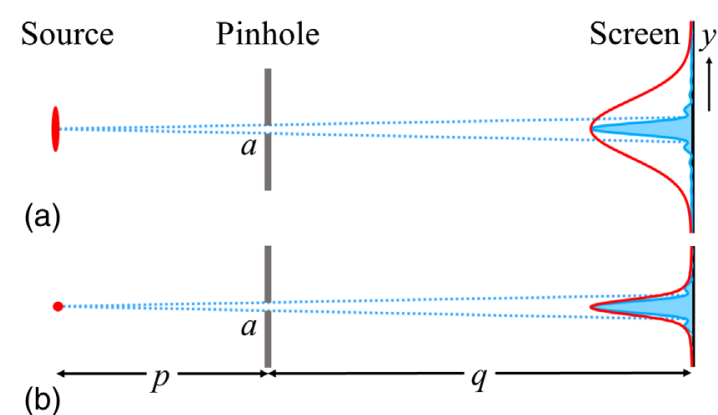

FIG. 1. Schematic of a pinhole imaging system. (a) shows a relatively large source size and its profile on the detector in red. The blue profile is the point spread function of the pinhole for a point source [shown in both (a) and (b) for reference]. (b) shows a small source that might be expected from an MBA-type lattice. Note that the source size effects are comparable to the point spread function.

detector system [6]. Assuming all contributions are Gaussian shape functions, the root mean square (rms) size of each contribution can be added in quadrature. The measured rms size of the image, $\Sigma$, is thus

$$
\Sigma^{2}=\left(M \sigma_{y}\right)^{2}+\sigma_{\text {pinhole }}^{2}+\sigma_{\text {detector }}^{2},
$$

where $\sigma_{y}$ is the electron source size, $M$ is the magnification factor $(M=q / p), \sigma_{\text {pinhole }}$ is the rms size of the pinhole PSF, and $\sigma_{\text {detector }}$ is the detector rms resolution that can be determined experimentally [7]. Using Eq. (1), the electron source size $\sigma_{y}$ can be extracted from the measured $\Sigma$, $\sigma_{\text {detector }}$, and calculated $\sigma_{\text {pinhole }}$.

The $\sigma_{\text {pinhole }}$ term can be estimated analytically as

$$
\sigma_{\text {pinhole }}^{2}=\sigma_{\text {geo }}^{2}+\sigma_{\text {diff }}^{2}
$$

where $\sigma_{\text {geo }}$ and $\sigma_{\text {diff }}$ are the size contribution from the geometric projection and diffraction of the pinhole, respectively.

Simplifying the discussion to one dimension, the geometric projection size of the pinhole (or 1D slit) is given by [8]

$$
\sigma_{\mathrm{geo}}=\frac{x_{g} a(p+q)}{p}=\frac{a}{2 \sqrt{3}} \frac{(p+q)}{p},
$$

where $a$ is the slit width and $p$ and $q$ are the source-topinhole and pinhole-to-detector distances, respectively. $x_{g}$ is a constant to scale the slit width $a$ to an equivalent rms value. Here we choose $x_{g}=1 / 2 \sqrt{3}$ so that $x_{g} a$ is the rms width of a rectangular shape with width $a$.

The diffraction profile of a single slit in the far-field approximation (Fraunhofer approximation) can be expressed analytically as [15]

$$
I(y)=I_{0} \operatorname{sinc}^{2}\left(\frac{\pi a}{\lambda q} y\right),
$$

where $I_{0}$ is the peak intensity, $y$ is the vertical coordinate in the detector plane (see Fig. 1), and $\lambda$ is the wavelength. The FWHM size of this diffraction profile is $0.886 \lambda q / a$. A factor of $1 / 2.355$ is used to convert from the FWHM to the rms value for a Gaussian function. The rms diffraction size of a pinhole (or a slit) is then

$$
\sigma_{\text {diff }}=\frac{\lambda q}{x_{d} a}=\frac{0.886}{2.355} \frac{\lambda q}{a}=\frac{\lambda q}{2.658 a},
$$

where $x_{d}$ is a constant.

\section{B. Design optimization}

The resolution of pinhole imaging is determined by the PSF of the pinhole and detector. The detector contribution needs to be as small as possible. The slit size can be optimized by minimizing the ratio of the pinhole PSF to the magnified source image size:

$$
\frac{\sigma_{\text {pinhole }}}{M \sigma_{y}} .
$$

The optimized slit size $a_{0}$ can be obtained by setting the derivative of Eq. (6) to zero [6], which gives

$$
a_{0}=\sqrt{\frac{\lambda q p}{x_{d} x_{g}(q+p)}}=\sqrt{\frac{0.767 \lambda q p}{q+p}} .
$$

Substituting Eq. (7) into Eq. (6), the minimized ratio can be obtained as

$$
\left(\frac{\sigma_{\text {pinhole }}}{M \sigma_{y}}\right)_{\min }=\frac{1}{\sigma_{y}} \sqrt{\frac{2 \lambda x_{g}}{x_{d}}\left(p+\frac{p^{2}}{q}\right)} .
$$

Equation (8) shows that the resolution of the system can be improved by choosing a short wavelength, small sourceto-pinhole distance, and large pinhole-to-detector distance.

The above analytical formulas based on the far-field approximation give many physical insights to the system and provide general design guidelines for pinhole imaging systems. However, a near-field numerical simulation is needed for obtaining accurate optimization parameters.

The PSF of the pinhole can be calculated from near-field (Fresnel) diffraction. The diffraction profile of a 1D slit at distance $q$ is given by [15]

$$
\begin{aligned}
I_{s}(y) & =\varepsilon(y) \cdot \varepsilon^{*}(y), \\
\varepsilon(y) & =\frac{1}{i \lambda q} \int_{-a / 2}^{a / 2} \varepsilon_{0}\left(y_{0}\right) \exp \left[\frac{i \pi}{\lambda q}\left(y-y_{0}\right)^{2}\right] d y_{0},
\end{aligned}
$$

where $\varepsilon_{0}\left(y_{0}\right)$ is the wave field in the pinhole plane. For a point source located at a distance $p$ upstream of the pinhole, the normalized wave field is given by 


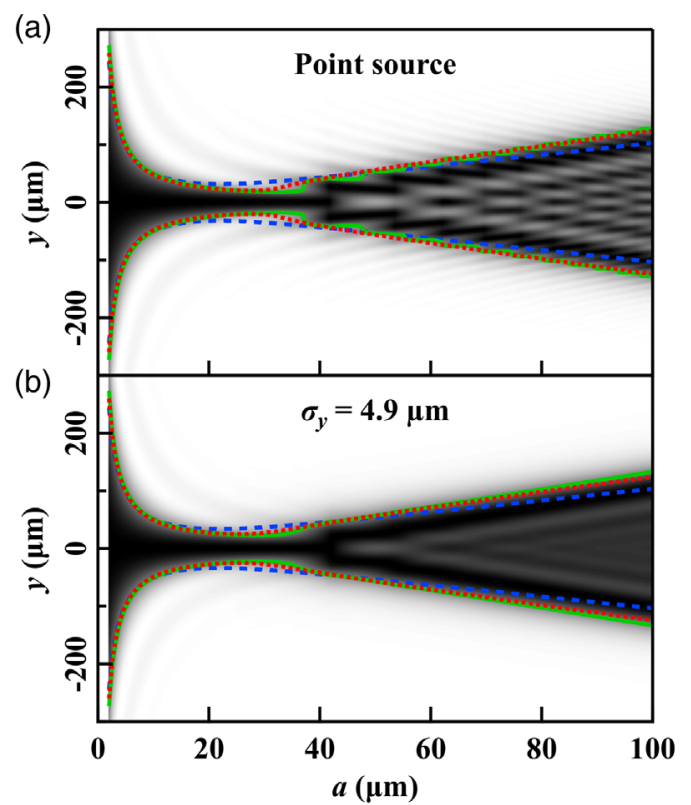

FIG. 2. Normalized diffraction profiles from a 1D slit with different $a$ sizes simulated with Eqs. (9)-(11) for (a) a point source and (b) a Gaussian source with size $\sigma_{y}=4.9 \mu \mathrm{m}$ (M3 bend magnet for the Advanced Photon Source upgrade source). The grayscale is linear to the profile intensity, and a darker color indicates a higher intensity. The calculation parameters are $\lambda=$ $0.827 \AA$ (photon energy, $E=15 \mathrm{keV}$ ), $p=6.6 \mathrm{~m}$, and $q=13.4 \mathrm{~m}$. The dashed curves are the $\pm \sigma_{\text {pinhole }}$ values obtained from Eq. (8), the solid curves are the FWHM/2.355 values of the diffraction profiles, and the dotted curves are the Gaussian fitted $\sigma$ values.

$\varepsilon_{0}\left(y_{0}\right)=\frac{p}{\sqrt{p^{2}+y_{0}^{2}}} \exp \left[\frac{i 2 \pi}{\lambda}\left(\sqrt{p^{2}+y_{0}^{2}}-p\right)\right]$

With a Gaussian distribution source, the intensity profile is given as a convolution of the diffraction pattern with the (de)magnified source profile:

$$
I_{G}=I_{s}(y) \otimes\left[\exp \left(-\frac{y^{2} p^{2}}{2 \sigma_{y}^{2} q^{2}}\right)\right]
$$

where the $\otimes$ symbol represents the convolution operation.

Examples of simulated diffraction profiles from a 1D slit are shown in Fig. 2 with parameters listed in the figure caption. To compare the analytical solution and the numerical calculation, Fig. 2 also shows the $\pm \sigma_{\text {pinhole }}$ values extracted with different methods: (i) (dashed curves) from analytical formula Eq. (8), (ii) (solid curves) from the FWHM/2.355 values of the Fresnel diffraction profiles calculated with Eqs. (9)-(11), and (iii) (dotted curves) from the Gaussian fitted $\sigma$ values of the diffraction profiles. Results from the analytical approach provide correct trends but tend to overestimate the minimum $\sigma_{\text {pinhole }}$.

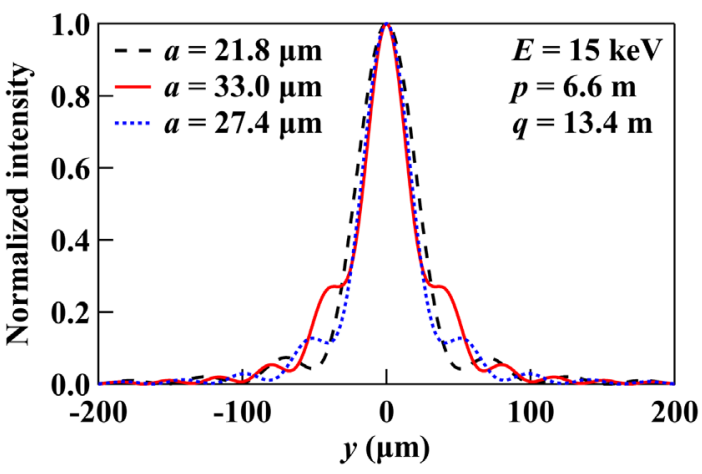

FIG. 3. Simulated PSF of a 1D slit with size $a_{0}$ obtained from (dashed curve) analytical formula Eq. (7), (solid curve) minimum profile width from the solid curves in Fig. 2(a), and (dotted curve) minimum Gaussian-fitted width from the dotted curves in Fig. 2(a).

Figure 3 shows PSF simulated with Eqs. (9)-(11) with the optimized slit size determined from the three methods above. The near-field diffraction methods give smaller $\sigma_{\text {pinhole }}$ compared to the analytical approach. The minimum $\sigma_{\text {pinhole }}$ is achieved when the diffraction profile (solid curve in Fig. 3) has two obvious shoulder peaks. However, the profile is far away from a Gaussian shape, which means an accurate PSF simulation and deconvolution is required in the data analysis process for extracting source sizes. The minimum Gaussian fitted $\sigma_{\text {pinhole }}$ value is a better choice for fast data analysis and especially for real-time monitor systems.

The minimum $\sigma_{\text {pinhole }} /\left(M \sigma_{y}\right)$ ratios for different source sizes are shown in Fig. 4. Optimized pinhole sizes $a$ to achieve a minimum $\sigma_{\text {pinhole }} /\left(M \sigma_{y}\right)$ and simulated image sizes $\Sigma$ are summarized in Table I for different source sizes. When the source size is small, the PSF of the pinhole $\sigma_{\text {pinhole }}$ is much larger than the magnified source image size $M \sigma_{y}$. Therefore, the pinhole imaging technique is normally considered not appropriate for small source size $(<10 \mu \mathrm{m})$

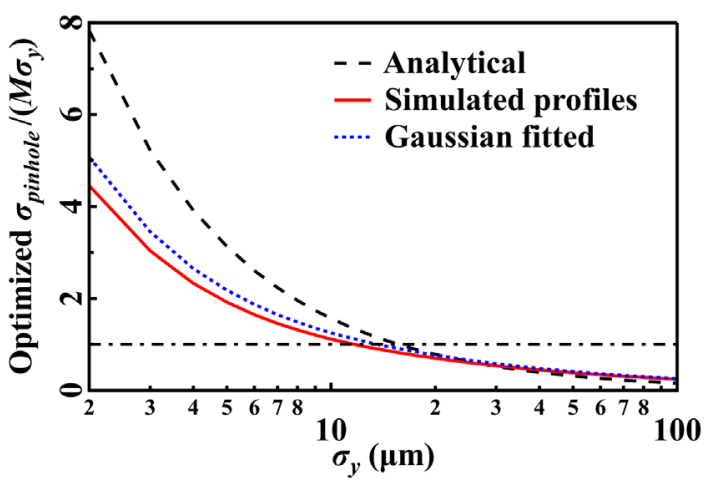

FIG. 4. Minimum $\sigma_{\text {pinhole }} /\left(M \sigma_{y}\right)$ for different source sizes $\sigma_{y}$ obtained from three methods; see the text for details. The dotdash line indicates $\sigma_{\text {pinhole }} /\left(M \sigma_{y}\right)=1$. 
TABLE I. Optimized pinhole sizes $a$ to achieve a minimum $\sigma_{\text {pinhole }} /\left(M \sigma_{y}\right)$ and simulated image sizes $\Sigma$ from the three methods for different source sizes $\sigma_{y}$.

\begin{tabular}{lccccccc}
\multicolumn{2}{c}{$\sigma_{y}(\mu \mathrm{m}) M \sigma_{y}(\mu \mathrm{m})$} & $a(\mu \mathrm{m}) \Sigma(\mu \mathrm{m})$ & $a(\mu \mathrm{m})$ & $\Sigma(\mu \mathrm{m})$ & $a(\mu \mathrm{m})$ & $\Sigma(\mu \mathrm{m})$ \\
\hline 0 & 0 & 21.8 & 27.0 & 32.9 & 15.1 & 27.3 & 17.2 \\
2 & 4 & 21.8 & 27.3 & 31.3 & 15.9 & 27.3 & 18.0 \\
4.9 & 9.8 & 21.8 & 28.8 & 28.9 & 19.3 & 25.7 & 21.2 \\
10 & 20 & 21.8 & 33.8 & 24.9 & 28.0 & 23.3 & 29.7 \\
20 & 40 & 21.8 & 48.8 & 22.5 & 47.2 & 22.5 & 48.5 \\
50 & 100 & 21.8 & 105.0 & 24.9 & 106.8 & 25.7 & 107.5 \\
\hline \hline
\end{tabular}

measurements. Table I also shows that the optimized pinhole size $a$ does not vary much for different source sizes.

Another main contribution to the instrument resolution is the PSF of the detector system, which has been studied previously [6,7]. The PSF of the detector is normally measured with a sharp edge absorber. In principle, the detector contribution needs to be as low as possible to be comparable to or smaller than $M \sigma_{y}$. The reported detector system, in existing pinhole monitors, consists of a scintillator screen and a visible light CCD camera. It can have a PSF $\sigma_{\text {detector }}$ as low as $6.2 \mu \mathrm{m} \mathrm{[7],} \mathrm{which} \mathrm{is} \mathrm{smaller} \mathrm{than} \mathrm{the}$ contribution of pinhole as shown in Table I. Therefore, the detector resolution is not the limiting factor for the pinhole imaging technique. However, it is still necessary to measure the detector resolution accurately in order to deconstruct the correct source size.

There are also practical limitations to the pinhole imaging technique. One challenge is the fabrication of high-quality high-aspect-ratio pinholes for hard $\mathrm{x}$ rays. Another limit for high-speed beam size monitoring is the low photon statistics due to the small pinhole sizes. To improve the photon collection efficiency and consequently the imaging resolution and speed, a coded aperture imaging technique [16] is a possible solution.

\section{Coded aperture imaging}

Beam size monitors based on coded aperture imaging have been developed and implemented at several facilities [16-20]. The system uses a coded aperture, which is normally an array of slits or pinholes with variable size and spacing, as the imaging optics. The coded aperture system allows the transmission of many more photons than a single pinhole. Also, the resolution of the coded aperture system can be comparatively higher because of the combined information harvested from multiple apertures. At the same time, the data analysis of the coded aperture imaging is much more complicated for the same reason. The two major analysis methods are template fitting and direct deconvolution. The former relies on the closest matching of the measured beam image to the precalculated patterns with different source sizes and positions. The direct reconstruction can be carried out by either the Fourier transform method or the correlation method. In general, the template fitting method is more accurate but computationally expensive and, thus, slower. Details of these methods can be found in Ref. [16] and references therein.

\section{DOUBLE-SLIT INTERFEROMETRY}

\section{A. System description}

The use of double-slit interferometry to measure the beam size in particle accelerators was first developed by Mitsuhashi at KEK in Japan [9]. Since then, this system has been used at many synchrotron facilities as a way to measure the electron beam sizes [10,11,21-26]. This system is mostly implemented in the visible light regime (wavelength of 400-600 $\mathrm{nm}$ ) at third-generation light sources because of (i) the availability of high-quality visible light optics and (ii) a relatively large coherence length of the visible light. Figure 5 shows the basic elements of a double-slit interferometer which consists of two narrow slits located at a distance $p$ downstream of the source and a detector system to measure the interference pattern at a distance $q$ downstream of the double slits.

A practical visible-light interferometer may contain more elements including beam extraction mirrors, focusing optics to reach the far-field condition, a polarizer to select one polarization, and a bandpass filter to obtain quasimonochromatic radiation. The performance of such a system highly relies on the quality of the optical components and the noise in the system. The main concern of the interferometer is the distortion of the wavefront due to aberration caused by the optics. Another limitation of the system is the distortion due to mechanical vibration and air fluctuations. The electron beam oscillation can also distort the interferogram, and, therefore, fast detection is needed for accurate measurements.

Recently, for use in new generation facilities with ultrasmall source sizes, new systems have been proposed and tested in the $\mathrm{x}$-ray regime ( $\sim 0.1 \mathrm{~nm}$ wavelengths) [27]. For x-ray interferometers, the expected resolution is much higher ( $\sim$ submicron). The setup is relatively simple and does not require as many optical components. At the shorter wavelength, a smaller slit size and slit separation are needed. For a typical hard x-ray fourth-generation

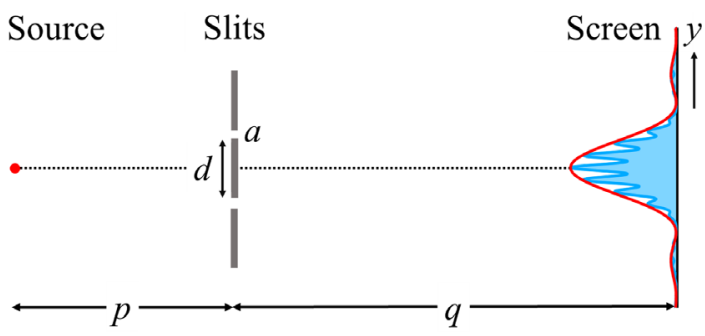

FIG. 5. Schematic of a double-slit interferometry system. 
synchrotron source, the electron beam size can be as low as a few microns.

The double-slit interference, for an extended source, can be described by an analytical intensity formula within the far-field (Fraunhofer) approximation at a wavelength $\lambda$ as [15]

$$
I=2 I_{0} \operatorname{sinc}^{2}\left(\frac{\pi a}{\lambda q} y\right)\left[1+V \cos \left(\frac{2 \pi d}{\lambda q} y\right)\right],
$$

where $a$ is the slit width, $d$ is the separation between the two slits, and $V$ is the complex degree of coherence. Equation (12) assumes the amplitudes of the wave field passing through the two slits are equal.

The van Cittert-Zernike theorem [28] states that, for an incoherent source, the complex degree of coherence at a distance from the source can be expressed as a Fourier transform of the spatial intensity distribution of the source. For a source with a spatially Gaussian distribution, the degree of coherence is also a Gaussian function given by

$$
V=\exp \left(-\frac{2 \pi^{2} d^{2} \sigma_{y}^{2}}{\lambda^{2} p^{2}}\right)
$$

Experimentally, $V$ is the visibility of the double-slit interference pattern and can be measured as

$$
V=\frac{I_{\max }-I_{\min }}{I_{\max }+I_{\min }}
$$

where $I_{\min }$ and $I_{\max }$ are the minimum and maximum intensities, respectively, of the interference pattern in the vicinity of the interferogram center. It should be noted that $0 \leq V \leq 1$.

Based on Eq. (13), the source size can be extracted from a single-point measurement of $V$ (one $V$ value at a fixed slit separation $d$ ) as

$$
\sigma_{y}=\frac{\lambda p}{\pi d} \sqrt{\frac{1}{2} \ln \frac{1}{V}}
$$

\section{B. Design optimization}

The resolution of the double-slit interferometer relies on the statistical error $d V$ in measuring fringe visibility. The relative size error $d \sigma_{y} / \sigma_{y}$ can be derived from Eq. (15) as

$$
\frac{d \sigma_{y}}{\sigma_{y}}=\frac{d V}{12 \ln \frac{1}{V}} .
$$

Note that the relative size error is only a function of visibility. For a visibility error of $d V=0.01$, the $V$ dependent size measurement error is plotted in Fig. 6.

To have a $5 \%$ or better sensitivity of source size measurement, or $d \sigma_{y} / \sigma_{y}<0.05$, the visibility range of

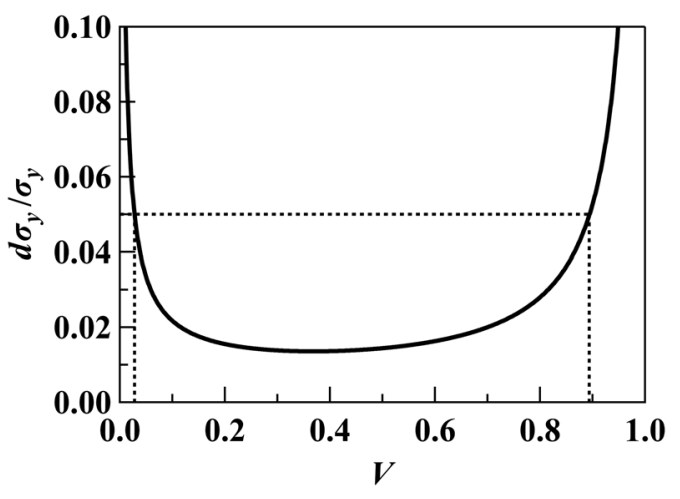

FIG. 6. Relative size measurement error as a function of fringe visibility $V$ for a 0.01 visibility uncertainty. The region between the vertical dotted lines is the visibility range that gives $d \sigma_{y} / \sigma_{y}<0.05$.

the system needs to be in the range of $0.028<V<0.894$ (see Fig. 6) with the minimum $d \sigma_{y} / \sigma_{y}$ at $V=0.368$. Then the detectable size range is $0.0753\left(\frac{\lambda p}{d}\right)<\sigma_{y}<0.426\left(\frac{\lambda p}{d}\right)$ based on Eq. (15). To have a $2 \%$ or better sensitivity of source size measurement, the visibility and detectable range becomes $0.12<V<0.70$ and $0.13\left(\frac{\lambda p}{d}\right)<\sigma_{y}<0.33\left(\frac{\lambda p}{d}\right)$, respectively. The optimized detectable source size is obtained from Eq. (15) with $V=0.368$ to be

$$
\sigma_{y}=0.225 \frac{\lambda p}{d}
$$

Figure 7 shows the detectable source size range with $2 \%$ sensitivity as a function of slit separation $d$. It provides a guideline for choosing the slit separation for the desired source size range. For a vertical source size of $4.9 \mu \mathrm{m}$ (M3 bend magnet for the Advanced Photon Source upgrade source), a slit separation of $25 \mu \mathrm{m}$ is optimum for $\lambda=$ $0.827 \AA(E=15 \mathrm{keV})$ and $p=6.6 \mathrm{~m}$.

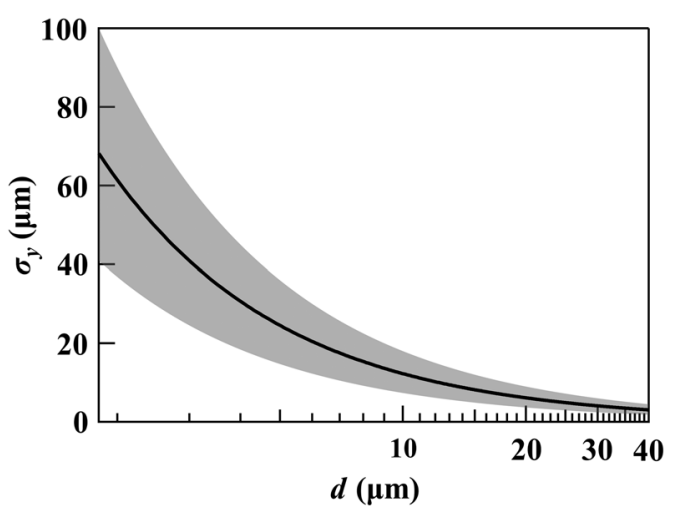

FIG. 7. Detectable source size range (gray area) as a function of slit separation $d$ for a 0.01 visibility uncertainty. The solid curve shows the optimized detectable source size given by Eq. (17) with $\lambda=0.827 \AA(E=15 \mathrm{keV})$ and $p=6.6 \mathrm{~m}$. 


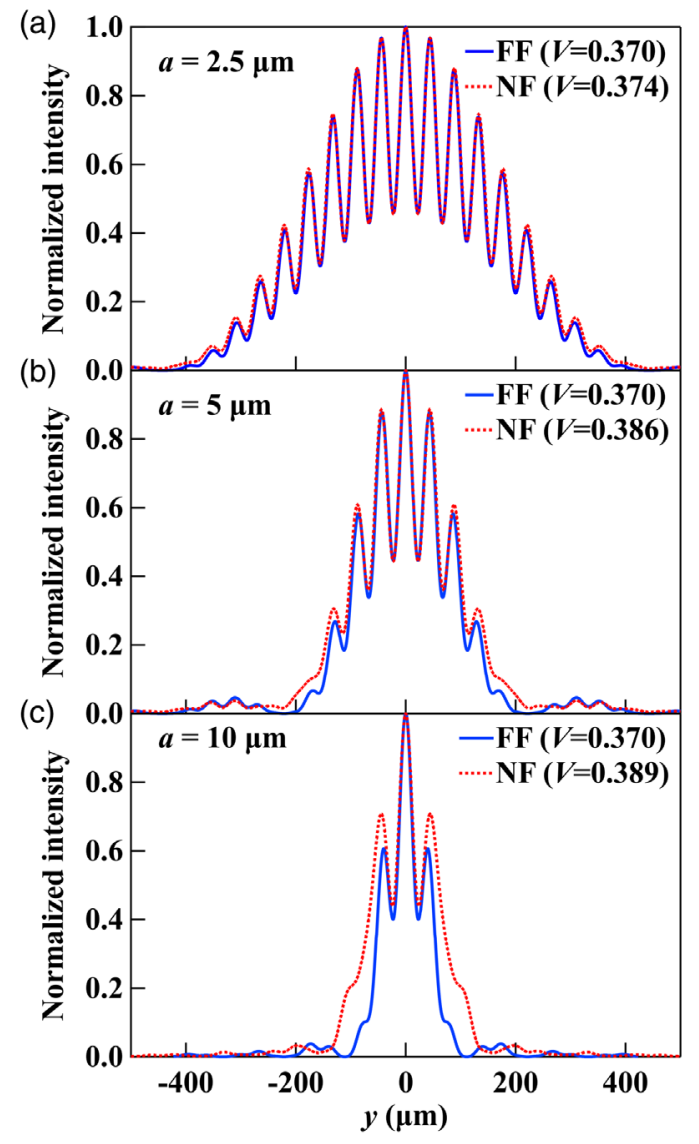

FIG. 8. Simulated double-slit interference profiles using (solid curves) far-field formula Eq. (12) and (dotted) near-field (Fresnel) calculation with different slit widths $a=$ (a) 2.5, (b) 5.0, and (c) $10 \mu \mathrm{m}$. The other calculation parameters are $\lambda=0.827 \AA$ $(E=15 \mathrm{keV}), \quad p=6.6 \mathrm{~m}, \quad q=13.4 \mathrm{~m}, \quad \sigma_{y}=4.9 \mu \mathrm{m}$, and $d=25 \mu \mathrm{m}$. The figure shows the failure of the far-field approximation for large slit width $a$.

The choice of slit width can be estimated from the farfield interference profile in Eq. (12). The peak width of the envelope function is given by the sinc function, while the period of the interference pattern is defined by the cos function in Eq. (12). The size of each single slit needs to be small enough so that there are enough double-slit interference peaks within the central envelope peak. When the slit width is large $(a>d / 5)$, the far-field approximation starts to fail, which causes further complications. Figure 8 compares double-slit interference profiles calculated by Eq. (12) and by near-field (Fresnel) simulation with different slit widths. A slit width of $a=d / 5$ is adequate for most cases.

Double-slit interferometry methods require a monochromatic photon beam. The choice of photon energy is flux driven. For a desired source size measurement, the optimized condition based on Eq. (17) requires that $(\lambda p / d)$ or $(p / E d)$ is a constant. Since slit width $a$ is proportional to $d$, then $p / E a$ is a constant. The total transmitted flux through a fixed size double slit is proportional to slit width $a$ and

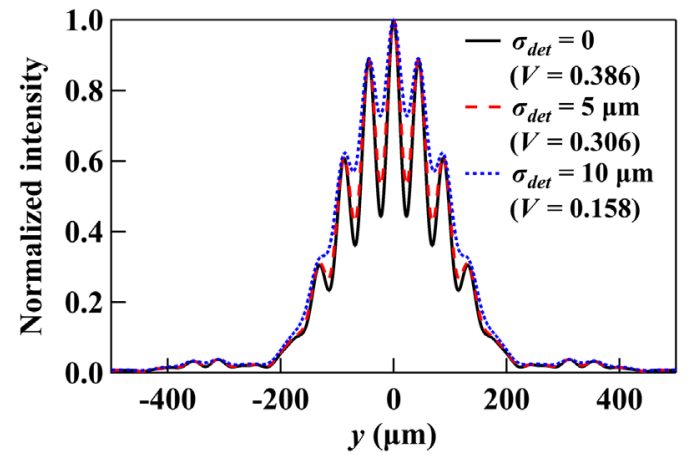

FIG. 9. Simulated double-slit interference profiles using nearfield (Fresnel) calculation with different detector resolutions $\sigma_{\text {det }}$ of a Gaussian PSF equals to 0 (solid curve), 5.0 (dashed curve), and $10 \mu \mathrm{m}$ (dotted curve). Other parameters are $\lambda=0.827 \AA$ $(E=15 \mathrm{keV}), \quad p=6.6 \mathrm{~m}, \quad q=13.4 \mathrm{~m}, \quad \sigma_{y}=4.9 \mu \mathrm{m}, \quad$ and $d=25 \mu \mathrm{m}$.

inversely proportional to $p^{2}$. Therefore, the total flux is proportional to $a / p^{2}$ or $1 / E p$. Considering that most of the scintillator detectors have linear responses in energy, the overall response on the detector is proportional to $1 / p$. Thus, the energy choice depends only on the flux spectrum of the BM radiation. Also, the source-to-slit distance $p$ needs to be minimized to maximize the flux. On the other hand, smaller $p$ and $\lambda$ implies smaller $d$ and $a$, which makes slit manufacturing difficult.

In summary, the energy should be chosen to optimize the flux; from there, an optimized slit separation and size are chosen based on the source size, and then $p$ needs to be as small as practically possible.

Detector resolution is also important for the double-slit interference method. Effects of detector resolution $\left(\sigma_{\operatorname{det}}\right.$ of a Gaussian PSF) on the interference pattern are shown in Fig. 9. The detector resolution needs to be high enough to maintain high visibility, and the detector PSF needs to be measured accurately to extract correct source sizes. The blurring effect of a detector is also determined by the peak separation in the interference pattern. Peak separation is the period of the cos function in Eq. (12), given by $\lambda q / d$. Since $\lambda / d$ is predetermined from the desired source size by Eq. (17), the only way to reduce the detector blurring is to increase the slit-to-detector distance $q$. However, increasing $q$ will reduce the flux density on the detector.

\section{C. $\pi$ polarization with diffraction obstacle}

The original $\pi$-polarization method is based on measuring the image of the $\pi$-polarized UV-vis light $[29,30]$. The filament-beam-spread function (FBSF) of the $\pi$ polarized UV-vis light has an on-axis zero intensity minimum. The recorded image at the detector is a convolution of the FBSF, the detector PSF, and the electron source distribution and, thus, has a blurred nonzero on-axis minimum. The electron source size can be extracted from 
the measured on-axis minimum when compared to precalculated values. The electron source profile can be obtained by deconvoluting the FBSF from the measured beam image. The $\pi$-polarization method relies heavily on the accuracy of the FBSF model. All existing systems use the synchrotron radiation workshop (SRW) [31] as the backbone simulation software.

To improve the resolution of the $\pi$-polarization method, diffraction obstacles with different sizes can be introduced to block the central part of the photon beam $[32,33]$. This so-called "obstacle diffractometer" is a variation of the double-slit interferometer. The method expects to have advantages over the traditional double-slit interferometry because of the higher acceptance angle and higher flux on the detector. The smallest electron beam size measured by the obstacle diffractometer was reported to be $3 \mu \mathrm{m}$ with less than $10 \%$ rms error [34].

\section{IV. pS-BPM SYSTEM}

\section{A. System description}

The ps-BPM system was recently developed at the Canadian Light Source [12,13]. The unique feature of the system is its ability to measure simultaneously four properties of the electron source: position, angle, size, and divergence in the vertical direction. Apart from its main application as a beam position monitor, the ps-BPM system was predicted to be able to measure source sizes of a few microns [14].

The ps-BPM system contains a crystal monochromator, a $\mathrm{K}$-edge filter, and a detector. The monochromator is tuned to the K-edge energy of a filter and provides an energydispersed photon beam across the vertical opening angle of the bend magnet radiation. This energy dispersion is spatially mapped on the detector. The measured K-edge width $\sigma_{\text {edge }}$ on the detector contains contributions from the natural energy width of the K edge $[35,36]$ projected to an angular width $\sigma_{y_{K \text {-edge }}^{\prime}}$, the angular bandwidth of the monochromator crystal [37], $\sigma_{y_{\text {mono }}^{\prime}}$, and the electron source size $\sigma_{y}$. Assuming all contributions are Gaussian shape functions, the electron source size can be obtained by

$$
\sigma_{y}=\sqrt{\sigma_{\text {edge }}^{2}-\left(D \sigma_{y_{\text {-edge }}^{\prime}}\right)^{2}-\left(D \sigma_{y_{\text {mono }}^{\prime}}\right)^{2}}
$$

where $D$ is the source-to-detector distance. The electron source divergence $\sigma_{y^{\prime}}$ can be extracted from the measured full vertical beam width $\sigma_{\text {beam }}$ without the filter by

$$
\sigma_{y^{\prime}}=\frac{1}{D} \sqrt{\sigma_{\text {beam }}^{2}-\sigma_{y}^{2}-\left(D \sigma_{y_{\mathrm{Ph}}^{\prime}}\right)^{2}}
$$

where $\sigma_{y_{\mathrm{ph}}^{\prime}}$ is the natural opening angle of the photon beam [38].

\section{B. Design optimization}

The optimization of the ps-BPM system for measuring source size was studied previously [14] by investigating different monochromator crystal reflections and monochromator geometries, K-edge filter energies and concentrations, source-to-detector distances, and detector parameters. The key results are summarized here to provide a full picture, followed by detailed studies on the anticipated signal-tonoise ratio (SNR) to help guide choices for a practical system.

For the monochromator, the Bragg case geometry (reflection) was selected due to its higher reflectivity and, thus, higher flux over the Laue case geometry (transmission), although more effort must be made on thermalmechanical design and stability of the monochromator. Also, the scatter from the monochromator will depend on the geometry, which should be considered as it may affect the system SNR.

The choice of low-index crystal reflections [i.e., $\mathrm{Si}$ $(1,1,1)]$ and elements with higher K-edge energy (e.g., barium K edge at $37.441 \mathrm{keV}$ ) as a filter will improve the resolution of the system by reducing the total contribution of the crystal bandwidth, $D \sigma_{y_{\text {mono }}^{\prime}}$, and filter edge width $D \sigma_{y_{K \text {-edge }}^{\prime}}$ in Eq. (18). This will define the Gaussian width $\sigma_{\text {IRF }}$ of the instrument resolution function (IRF), $f_{\text {IRF }}(y)$, of the system as the combination of the spatial width associated with the monochromator dispersion and the K-edge width:

$$
\sigma_{\mathrm{IRF}}=\sqrt{\left(D \sigma_{y_{\text {mono }}^{\prime}}\right)^{2}+\left(D \sigma_{y_{K \text {-dge }}^{\prime}}\right)^{2}} .
$$

Based on Eq. (20), the resolution of the system to measure the source size will improve as a function of $1 / D^{2}$; thus, a small source-to-detector distance is preferred. A distance of $D=10 \mathrm{~m}$ is a practical choice given the limitations of storage ring design and a beam line for psBPM measurements.

With the prior considerations regarding choices of layout and optics, ultimately the resolution of the ps-BPM system will rely on the photon flux and noise level (SNR), which will now be considered.

A simplified model is described below to study the SNR requirement for measuring different source sizes. A Gaussian function is generated to represent the normalized beam profile with

$$
I_{\text {beam }}(y)=e^{-\left(y^{2} / 2 \sigma_{\text {beam }}^{2}\right)},
$$

where $y$ is the vertical position in the detector plane with $\sigma_{\text {beam }}$ being the Gaussian width of the beam profile on the detector. The filtered beam profile is obtained from multiplying $I_{\text {beam }}$ by the transmission function of the filter

$$
I_{\text {filtered }}(y)=I_{\text {beam }}(y) e^{-(\mu / \rho)(E) \rho t},
$$


where $\mu / \rho(E)$ is the mass attenuation coefficient as a function of photon energy $E$ around the K-edge energy $E_{k}$ of the filter, $\rho$ is the concentration, and $t$ is the thickness of the filter. Because of the dispersion effects of the crystal, $\mu / \rho(E)$ can be projected into a function of $y$ through

$$
E=\frac{E_{k}}{D \tan \theta_{B}} y,
$$

where $\theta_{B}$ is the Bragg angle of the monochromator crystal. The $\mu / \rho(y)$ function is related to the measured edge profile function $f_{\text {edge }}(y)$ through

$$
f_{\text {edge }}(y)=\frac{d\left\{-\ln \left[I_{\text {filtered }}(y) / I_{\text {beam }}(y)\right]\right\}}{d y}=\frac{d \frac{\mu}{\rho}(y)}{d y} \rho t
$$

The edge profile $f_{\text {edge }}(y)$ is a convolution of the IRF, $f_{\mathrm{IRF}}(y)$, and the electron source profile. The widths of these profiles follow the relationship described by Eq. (18) assuming all are Gaussian functions, or

$$
f_{\text {edge }}(y)=A e^{-\left(y^{2} / 2 \sigma_{\text {edge }}^{2}\right)}=A e^{-\left[y^{2} / 2\left(\sigma_{y}^{2}+\sigma_{\mathrm{IRF}}^{2}\right)\right]},
$$

where $A$ is a scaling parameter to match the edge jump of $\mu / \rho(E)$ above and below the K-edge energy for a selected filter element and filter thickness.

The sensitivity of the source size measurement is then carried out by adding noise to the calculated beam profiles [see Fig. 10(a)]. The ideal beam profile $I_{\text {beam }}(y)$ [see the

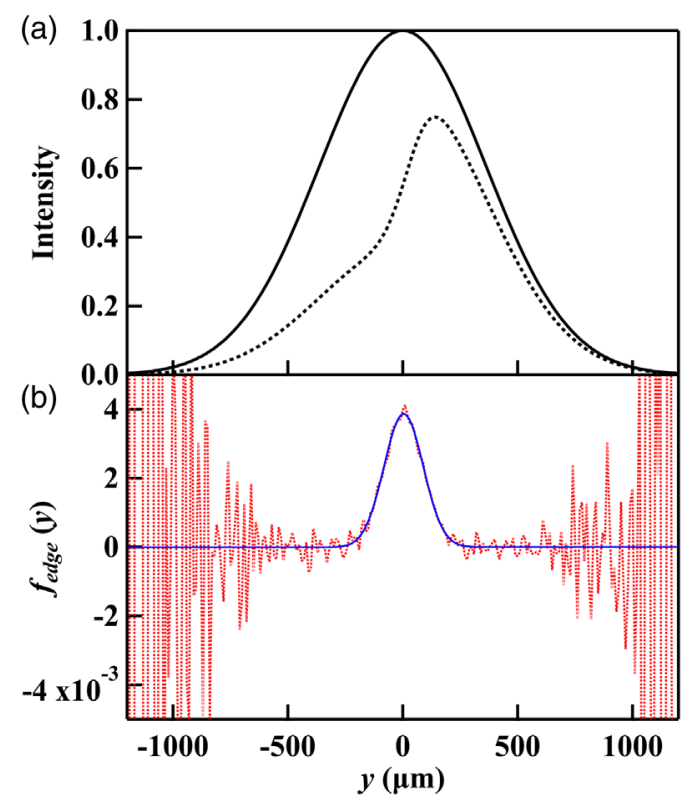

FIG. 10. (a) Calculated beam profiles $I_{\text {beam }}(y)$ (solid curve) and $I_{\text {filtered }}(y)$ (dotted curve) using Eqs. (21) and (24), respectively, with $\sigma_{y}=4.9 \mu \mathrm{m}, \sigma_{y}^{\prime}=2.8 \mu \mathrm{rad}, \sigma_{y_{\mathrm{Ph}}^{\prime}}=36.1 \mu \mathrm{rad}, \sigma_{D}=10 \mu \mathrm{m}$, and $\sigma_{\text {IRF }}=85 \mu \mathrm{m}$. (b) Extracted edge profiles $f_{\text {edge }}(y)$ with (dotted curves) and without (solid curves) added noise to the beam profiles in (a). solid line in Fig. 10(a)] is generated with Eq. (21) directly. The Gaussian edge profile is first generated with Eq. (25), integrated to give the $\mu / \rho(y)$ function using Eq. (24), and then substituted into Eq. (22) to form the ideal filtered profile $I_{\text {filtered }}(y)$ [see the dotted line in Fig. 10(a)]. Note that the step size in $y$ for all these profiles is chosen to represent the pixel size of the detector, $\sigma_{D}$.

Taking the M3 bend magnet source planned for the Advanced Photon Source upgrade [4] as an example, the nominal source parameters are $\sigma_{y}=4.9 \mu \mathrm{m}, \sigma_{y}^{\prime}=2.8 \mu \mathrm{rad}$, and $\sigma_{y_{\mathrm{Ph}}^{\prime}}=36.1 \mu \mathrm{rad}$. The width of the IRF for a Si $(1,1,1)$ Bragg single-crystal monochromator at the barium $\mathrm{K}$ edge $\left(E_{k}=37.441 \mathrm{keV}\right)$ is $\sigma_{\mathrm{IRF}}=85 \mu \mathrm{m}$ at a source-to-detector distance $D=10 \mathrm{~m} \mathrm{[14]}$.

If there is no noise added to the data, the edge profile can be extracted based on Eq. (24) and shown as the solid curve in Fig. 10(b). When a Gaussian random noise with a sigma size of 1/SNR is added to both filtered and unfiltered beam profiles, the extracted edge profile is shown as the dotted curve in Fig. 10(b). The fitted Gaussian width $\sigma_{\text {edge }}$ of the extracted edge profile from data with added noise is then used to extract the electron source size $\sigma_{y}$, using Eq. (18).

The extracted $\sigma_{y}$ values are evaluated with different input $\sigma_{y}$ values, detector pixel sizes $\sigma_{D}$, and SNR values. The goal is to find the minimum required SNR to ensure a source size measurement sensitivity $<5 \%$ of the source size. The standard deviation of 1000 independently extracted $\sigma_{y}$ values is used as a measure of sensitivity.

Figure 11(a) shows the required SNR to measure different source sizes $(1.25,2.5,5,10,20,40$, and $80 \mu \mathrm{m})$ with $5 \%$

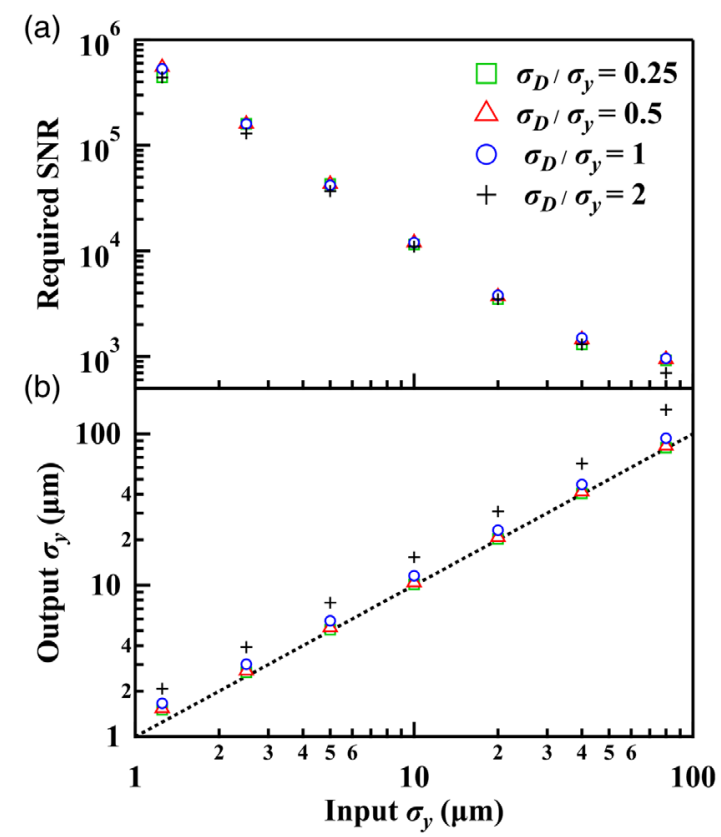

FIG. 11. (a) Required SNR to measure different source sizes with $5 \%$ precision and (b) the extracted electron source sizes for different input size values $\sigma_{y}$ and detector pixel sizes $\sigma_{D}$. 
sensitivity. To be able to measure a source size of $5 \mu \mathrm{m}$, an SNR of $4 \times 10^{4}$ is needed. The required SNR is proportional to $1 / \sigma_{y}^{2}$ and almost independent of the detector pixel size.

On the other hand, Fig. 11(b) shows that the extracted source size tends to be larger than the real value and increases as the detector pixel size increases. A pixel size comparable to source size $\sigma_{y}$ is enough to provide adequate accuracy for source sizes as small as $5 \mu \mathrm{m}$. In practice, the pixel size can be even larger considering that the IRF can be calibrated to include the effects of the detector pixel size. The IRF can be obtained either from a single measurement with a known source size or from a series of measurements by varying relative source sizes in a known ratio as shown in Ref. [13]. Also, the IRF can be obtained by comparing it with other measurement methods (e.g., pinhole imaging and double-slit interferometry).

The measurement noise of the ps-BPM system is determined by three main factors: dark noise of the detector, Compton scattering $[39,40]$ from the monochromator crystals, and fluorescence from the K-edge filter.

The dark noise of the detector determines the highest SNR of a single image in the absence of other noise sources. A good scientific complementary metal-oxidesemiconductor (sCMOS) camera can have a maximum dynamical range of a few tens of thousands [41].

The Compton scattering from the monochromator crystal depends on the crystal geometry, energy, angle, distances, and the spectral content of the incident SR beam. Calculations of the Compton scattering are compared against the signal which are either the monochromatic beam or filtered beam values. Figure 12 shows the ratio of the signal to the Compton background noise (SNR) with either a single Bragg or a single Laue monochromator geometry with different source-to-crystal distances at a

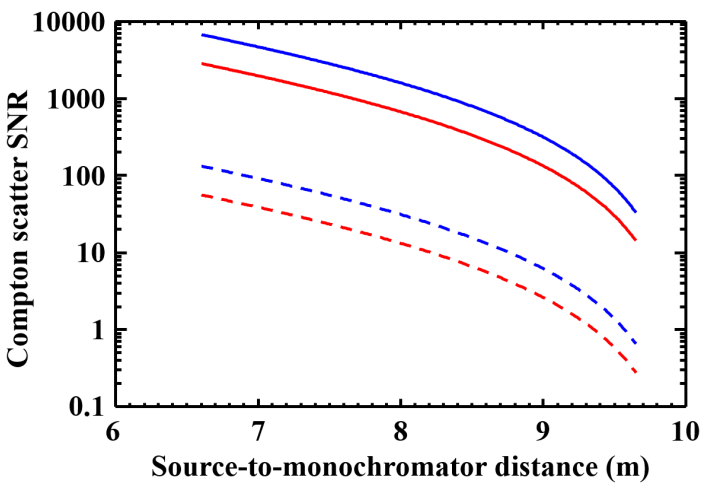

FIG. 12. Calculated Compton scatter SNR as a function of the source-to-monochromator distance with a fixed source-todetector distance for a single Bragg (solid curves) and a single Laue Si $(1,1,1)$ monochromator (dashed curves). The blue curves are for the unfiltered beam, and the red curves are for the filtered beam. Calculations are performed with storage ring energy $E_{\text {ring }}=6.0 \mathrm{GeV}$, storage ring current $I_{\text {ring }}=0.2 \mathrm{~A}$, and the bend magnet field $B_{0}=0.657 \mathrm{~T}$. fixed source-to-detector distance $(10 \mathrm{~m})$. The Bragg geometry shows a much higher SNR comparing to the Laue geometry at the minimum source to monochromator possible distance for APS-U $(6.6 \mathrm{~m})$ with the Bragg SNR 6700 and Laue SNR 130. Clearly, the Bragg geometry is preferred for a single crystal monochromator. This ratio of SNR is preserved for the edge side (Bragg SNR 2800, Laue SNR 50) but is reduced due to the filter absorption.

The fluorescence from the K-edge filter is another potential source of background noise; however, the estimation of its contribution was found to be insignificant compared to the Compton scatter.

In order to further reduce the Compton scattering background, a double-Bragg or a Laue-Bragg crystal type monochromator may be used, as it removes the line of sight between the detector and the first crystal which intercepts the entire SR beam and is the major source of scattering. The first crystal in a double-crystal monochromator being a Laue case has the advantage of simpler heat load management, which can reduce measurement errors caused by thermal deformation and drift.

The ps-BPM measures the vertical beam profile over a large horizontal BM fan. By summing up profiles over $N_{h}$ pixels in the horizontal direction, the SNR of the vertical profile measurement can be improved by a factor of $\sqrt{N_{h}}$ assuming the horizontal beam is uniform. By averaging over $N_{i}$ images, the SNR can be further improved to a factor of $\sqrt{N_{h} N_{i}}$. For example, from Fig. 11 the required SNR for measuring a $1.25 \mu \mathrm{m}$ source size is approximately $6 \times 10^{5}$. The Compton SNR will need to be improved by a factor of $\sqrt{N_{h} N_{i}}=90$ for a single Bragg monochromator (SNR 6700). Assuming $N_{h}$ is 1000 horizontal pixels, then $N_{i} \sim 8$ measurements are needed to achieve the required SNR of $6 \times 10^{5}$.

\section{CONCLUSION}

Three radiation-based methods of measuring electron source size were studied in this work. The optimization of each method was discussed in detail for measuring small source sizes at low-emittance synchrotron light sources.

A direct comparison of these methods is difficult, since each of them has its specific advantages and limitations and can provide complementary information about the source.

The pinhole imaging technique has the simplest setup and is free from optical aberration. The resolution of the technique relies heavily on the accurate modeling of the shape of the pinhole PSF and requires a high-resolution detector system. The pinhole imaging technique is compatible with a white beam and, thus, can provide relatively fast measurements.

The double-slit interferometry technique has the highest resolution using $\mathrm{x}$ rays. However, the detectable size range for a fixed slit separation is highly limited, especially for measuring small source sizes. A system with multiple or 
tunable slit separations will be needed to cover a large measurable range.

Both pinhole imaging and double-slit interferometry methods require the accurate near-field modeling of the PSF of the system. Also, the detector PSF plays a very important role in both methods.

The ps-BPM system is unique in terms of providing source information on the position, angle, size, and divergence simultaneously. Its source size measurement sensitivity is determined by the signal-to-noise ratio which requires careful system optimization to reduce scattering background. The ps-BPM system does not require a highresolution detector, and the knowledge of the detector PSF is not required. A Gaussian IRF model for the monochromator and K-edge contributions is adequate for data analysis.

At planned low-emittance storage rings, at least a dedicated BM beam line for beam diagnostic is a must. A combination of all three techniques will be ideal to provide a full characterization of the source. The pinhole imaging technique gives real-time monitoring of source profiles in both transverse directions. The double-slit interferometry can be used in either direction and can provide accurate source size measurement and calibration for pinhole imaging and the ps-BPM system. The ps-BPM system monitors all four parameters of the source in the vertical direction. A separate horizontal deflecting monochromator can be used to measure the horizontal source position and size.

\section{ACKNOWLEDGMENTS}

The authors acknowledge the financial support of Natural Sciences and Engineering Research Council of Canada (NSERC) Discovery Grant, Canadian Institutes of Health Research (CIHR) Team Grant-Synchrotron Medical Imaging, CIHR Training Grant-Training in Health Research Using Synchrotron Techniques, Canada Research Chair Program, Saskatchewan Health Research Foundation Team Grant, and the University of Saskatchewan. The research described in this paper was performed at the Canadian Light Source, which is funded by the Canada Foundation for Innovation, NSERC, the National Research Council Canada, CIHR, the Government of Saskatchewan, Western Economic Diversification Canada, and the University of Saskatchewan. This work was also supported by the U.S. Department of Energy, Office of Basic Energy Sciences, under Contract No. DEAC02-06CH11357.

[1] J. Buon, Beam phase space and emittance, in CAS - CERN Accelerator School: 5th General Accelerator Physics Course, Jyväskylä, Finland, 1992, pp. 89-116, https:// doi.org/10.5170/CERN-1994-001.89.
[2] D. Einfeld, M. Plesko, and J. Schaper, First multi-bend achromat lattice consideration, J. Synchrotron Radiat. 21, 856 (2014).

[3] P. F. Tavares, S. C. Leemann, M. Sjöström, and Å. Andersson, The MAX IV storage ring project, J. Synchrotron Radiat. 21, 862 (2014).

[4] M. Borland et al., The upgrade of the Advanced Photon Source, in Proceedings of the 9th International Particle Accelerator Conference (IPAC2018), Vancouver, BC, Canada (JACoW, Geneva, Switzerland, 2018), pp. 2872-2877, https://doi.org/10.18429/JACoW-IPAC2018-THXGBD1.

[5] G. Kube, Review of synchrotron radiation based diagnostics for transverse profile measurements, in Proceedings of the 8th European Workshop on Beam Diagnostics and Instrumentation for Particle Accelerators (DIPAC 2007), Venice, Italy (Elettra Sincrotrone, Trieste, Italy, 2007), pp. 6-10.

[6] C. Thomas, G. Rehm, I. Martin, and R. Bartolini, X-ray pinhole camera resolution and emittance measurement, Phys. Rev. ST Accel. Beams 13, 1 (2010).

[7] C. A. Thomas and G. Rehm, Pinhole camera resolution and emittance measurement, in Proceedings of the 11th European Particle Accelerator Conference, Genoa, 2008 (EPS-AG, Genoa, Italy, 2008), pp. 1254-1256.

[8] P. Elleaume, C. Fortgang, C. Penel, and E. Tarazona, Measuring beam sizes and ultra-small electron emittances using an X-ray pinhole camera, J. Synchrotron Radiat. 2, 209 (1995).

[9] T. Mitsuhashi, Beam profile and size measurement by SR interferometers, in Beam Measurement: Proceedings of the Joint US-CERN-Japan-Russia School on Particle Accelerators, Montreux and Geneva, Switzerland (World Scientific, Singapore, 1999), pp. 399-427, https://doi.org/ 10.1142/9789812818003_0018.

[10] T. Naito and T. Mitsuhashi, Very small beam-size measurement by a reflective synchrotron radiation interferometer, Phys. Rev. ST Accel. Beams 9, 122802 (2006).

[11] J. Corbett et al., Transverse beam profiling and vertical emittance control with a double-slit stellar interferometer, in Proceedings of the 5th International Beam Instrumentation Conference (IBIC 2016), Barcelona, Spain (JACoW, Geneva, Switzerland, 2016), pp. 236-239, https://doi.org/ 10.18429/JACoW-IBIC2016-MOPG70.

[12] N. Samadi, B. Bassey, M. Martinson, G. Belev, L. Dallin, M. de Jong, and D. Chapman, A phase-space beam position monitor for synchrotron radiation, J. Synchrotron Radiat. 22, 946 (2015).

[13] N. Samadi, X. Shi, L. Dallin, and D. Chapman, A real-time phase-space beam emittance monitoring system, J. Synchrotron Radiat. 26, 1213 (2019).

[14] N. Samadi, X. Shi, and D. Chapman, Optimization of a phase-space beam position and size monitor for lowemittance light sources, J. Synchrotron Radiat. 26, 1863 (2019).

[15] J. W. Goodman, Introduction to Fourier Optics (McGrawHill, New York, 1996).

[16] J. W. Flanagan, Review of recent status of coded aperture x-ray monitors for beam size measurement, in Proceedings of the 7th International Beam Instrumentation Conference (IBIC2018), Shanghai, China (JACoW, 
Geneva, Switzerland, 2018, pp. 361-365, https://doi.org/ 10.18429/JACoW-IBIC2018-WEOC02.

[17] C. Bloomer, G. Rehm, and J. W. Flanagan, Measurements of small vertical beamsize using a coded aperture at diamond light source, in Proceedings of the 3rd International Beam Instrumentation Conference (IBIC2014), Monterey, CA, USA (JACoW, Geneva, Switzerland, 2014), pp. 279-283.

[18] J. W. Flanagan, M. Arinaga, H. Fukuma, H. Ikeda, T. Mitsuhashi, and G. S. Varner, First measurements with coded aperture X-ray monitor at the ATF2 extraction line, in Proceedings of the 1st International Beam Instrumentation Conference (IBIC2012), 2013, pp. 237-240.

[19] J. P. Alexander et al., Operation of the CESR-TA vertical beam size monitor at $\mathrm{Eb}=4 \mathrm{GeV}$, Nucl. Instrum. Methods Phys. Res., Sect. A 798, 127 (2015).

[20] E. Mulyani, J. Flanagan, H. Fukuma, H. Ikeda, and M. Tobiyama, Image reconstruction technique based on coded aperture imaging for SuperKEKB x-ray beam size monitor, in Proceedings of the 9th International Particle Accelerator Conference (IPAC2018), Vancouver, BC, Canada, (JACoW, Geneva, Switzerland, 2018), pp. 4819-4822, https://doi.org/10.18429/JACoW-IPAC2018-THPML074.

[21] M. Masaki and S. Takano, Two-dimensional visible synchrotron light interferometry for transverse beam-profile measurement at the SPring-8 storage ring, J. Synchrotron Radiat. 10, 295 (2003).

[22] S. T. Wang, D. L. Rubin, J. Conway, M. Palmer, D. Hartill, R. Campbell, and R. Holtzapple, Visible-light beam size monitors using synchrotron radiation at CESR, Nucl. Instrum. Methods Phys. Res., Sect. A 703, 80 (2013).

[23] M. Koopmans et al., Applications of the interferometric beam size monitor at BESSY II, in Proceedings of the 9th International Particle Accelerator Conference (IPAC2018), Vancouver, BC, Canada (JACoW, Geneva, Switzerland, 2018), pp. 2103-2106, https://doi.org/10.18429/JACoWIPAC2018-WEPAK009.

[24] L. Torino and U. Iriso, Transverse beam profile reconstruction using synchrotron radiation interferometry, Phys. Rev. Accel. Beams 19, 122801 (2016).

[25] G. Trad, E. Bravin, A. Goldblatt, S. Mazzoni, F. Roncarolo, and T. Mitsuhashi, Beam size measurements using interferometry at LHC, in Proceedings of the 5th International Beam Instrumentation Conference (IBIC 2016), Barcelona, Spain (JACoW, Geneva, Switzerland, 2016, pp. 583-588, https://doi.org/10.18429/JACoW-IBIC2016WEBL02.

[26] M. L. Chen et al., Recent beam size measurement result using synchrotron radiation inteferometer in TPS, in Proceedings of the 5th International Beam Instrumentation Conference (IBIC 2016), Barcelona, Spain (JACoW,
Geneva, Switzerland, 2016), pp. 217-220, https:// doi.org/10.18429/JACoW-IBIC2016-MOPG63.

[27] W. Leitenberger, H. Wendrock, L. Bischoff, and T. Weitkamp, Pinhole interferometry with coherent hard X-rays, J. Synchrotron Radiat. 11, 190 (2004).

[28] M. Born and E. Wolf, Principles of Optics, 7th ed. (Cambridge University Press, Cambridge, England, 1999).

[29] A. Hansson, E. Wallén, and A. Andersson, Transverse electron beam imaging system using visible synchrotron radiation at MAX III, Nucl. Instrum. Methods Phys. Res., Sect. A 671, 94 (2012).

[30] Å. Andersson, M. Böge, A. Lüdeke, V. Schlott, and A. Streun, Determination of a small vertical electron beam profile and emittance at the Swiss Light Source, Nucl. Instrum. Methods Phys. Res., Sect. A 591, 437 (2008).

[31] O. Chubar and P. Elleaume, Accurate and efficient computation of synchrotron radiation in the near field region, in Proceedings of the 6th European Particle Accelerator Conference, Stockholm, 1998 (IOP, London, 1998), pp. 1177-1179.

[32] J. Breunlin, Å. Andersson, N. Milas, Á. Saá Hernández, and V. Schlott, Methods for measuring sub-pmrad vertical emittance at the Swiss Light Source, Nucl. Instrum. Methods Phys. Res., Sect. A 803, 55 (2015).

[33] J. Breunlin and $\AA$. Andersson, Emittance diagnostics at the MAX IV $3 \mathrm{GeV}$ storage ring, in Proceedings of the Seventh International Particle Accelerator Conference (IPAC2016), Busan, Korea (JACoW, Geneva, Switzerland, 2016), pp. 2908-2910, https://doi.org/10.18429/JACoWIPAC2016-WEPOW034.

[34] J. Breunlin, Å. Andersson, N. Milas, Saá Hernández, and V. Schlott, Methods for measuring sub-pm rad vertical emittance at the Swiss Light Source, Nucl. Instrum. Methods Phys. Res., Sect. A 803, 55 (2015).

[35] Y. A. Babanov, A. V. Ryazhkin, A. F. Sidorenko, and L. A. Blaginina, Correcting an experimental absorption spectrum for the core level width, J. Struct. Chem. 39, 833 (1998).

[36] O. Keski-Rahkonen and M. O. Krause, Total and partial atomic-level widths, At. Data Nucl. Data Tables 14, 139 (1974).

[37] W. H. W. Zachariasen, Theory of X-Ray Diffraction in Crystals (Wiley, New York, 1945).

[38] J. Schwinger, On the classical radiation of accelerated electrons, Phys. Rev. 75, 1912 (1949).

[39] A. H. Compton, A quantum theory of the scattering of x-rays by light elements, Phys. Rev. 21, 483 (1923).

[40] A. H. Compton, The spectrum of scattered x-rays, Phys. Rev. 22, 409 (1923).

[41] Andor, EMCCD vs sCMOS Cameras A Comparison available at https://www.oxinst.com/learning/view/article/ comparing-scmos. 